\title{
Can artificial intelligence help to detect dysplasia in patients with ulcerative colitis?
}

A 72-year-old man with an 18-year history of pancolitis had been visiting our hospital regularly for 12 years. He was taking oral mesalamine and mercaptopurine to sustain clinical remission. Surveillance colonoscopy was performed using high-definition endoscopy (CFHQ290ZI; Olympus, Tokyo, Japan) with an artificial intelligence (AI)-based detection system (EndoBRAIN-EYE; Cybernet Systems, Tokyo, Japan).

The Al-based detection system identified two lesions in the sigmoid colon ( $\vee$ Fig. 1 a,b) and indicated them with bounding boxes ( $>$ Video 1 )

Histological examination of the biopsy specimens showed that both of the lesions were characterized by low-grade dysplasia ( $\triangleright$ Fig. 2 a, b).

Patients with longstanding ulcerative colitis (UC) have a higher risk of colorectal cancer than do individuals in the general population [1]. UC-associated dysplasia is often flat with an unclear boundary from the surrounding tissues, making it difficult to detect [2]. Al-based polyp detection systems are used during colonoscopy to increase lesion detection [3]. The EndoBRAIN-EYE system can reportedly identify colorectal lesions with high accuracy in non-UC patients $[4,5]$. However, its use for the detection of dysplasia in patients with UC has not been previously reported.

With a target biopsy strategy in UC surveillance, the ability to detect lesions depends on the endoscopist. Al has the potential to help non-expert endoscopists detect dysplasia in patients with UC.

Endoscopy_UCTN_Code_TTT_1AQ_2AB
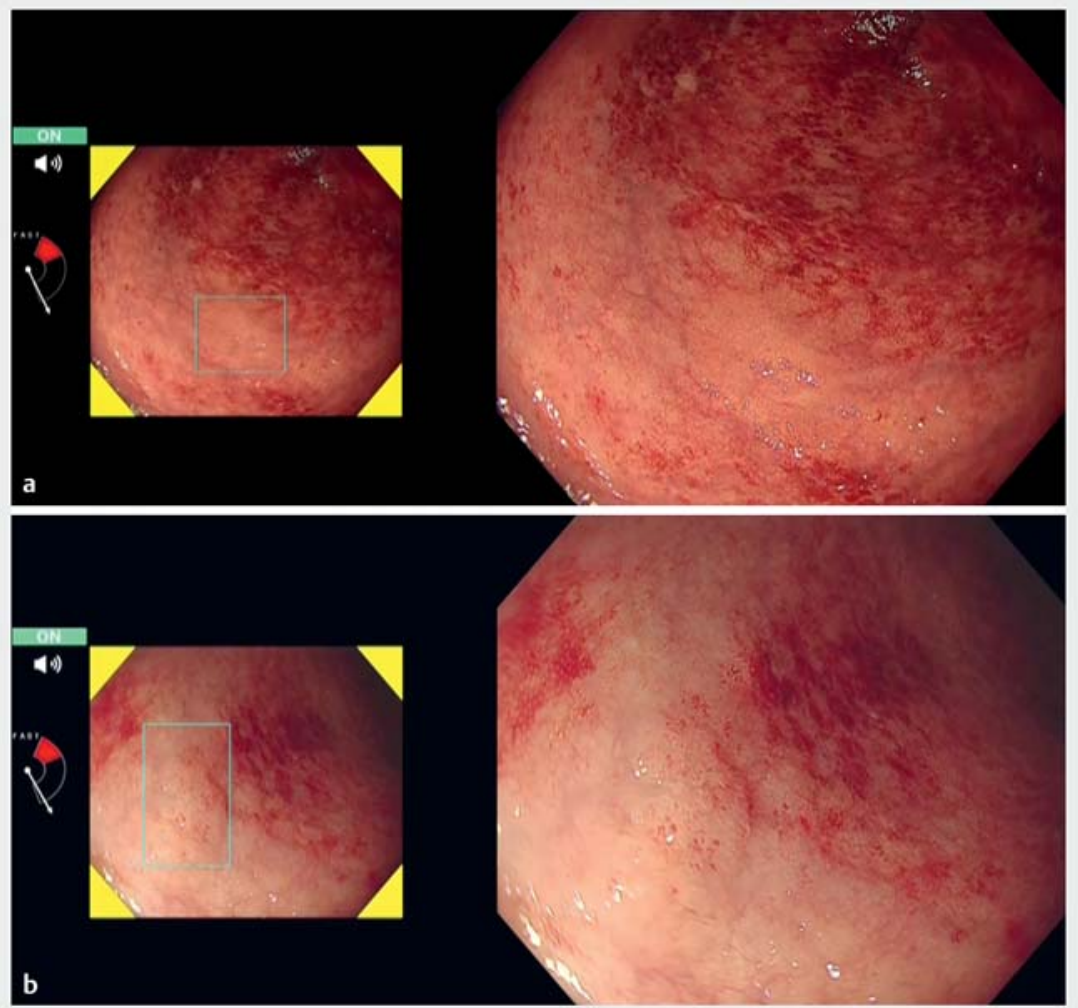

Fig. 1 The artificial intelligence-based detection system identified dysplasia. a First lesion. b Second lesion.

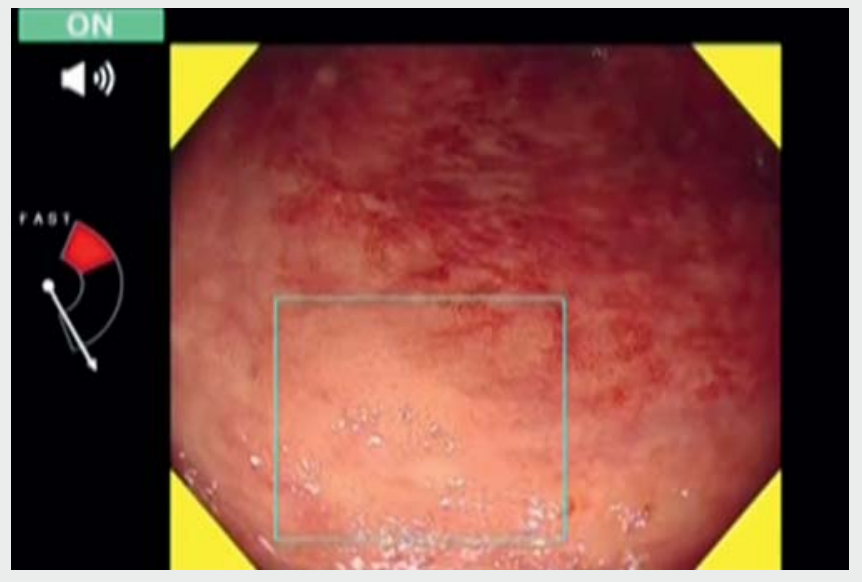

Video 1 At the moment the artificial intelligence-based detection system identified the dysplasia, it displayed the four corners of the monitor in yellow and indicated boxes bounding the dysplasia. 

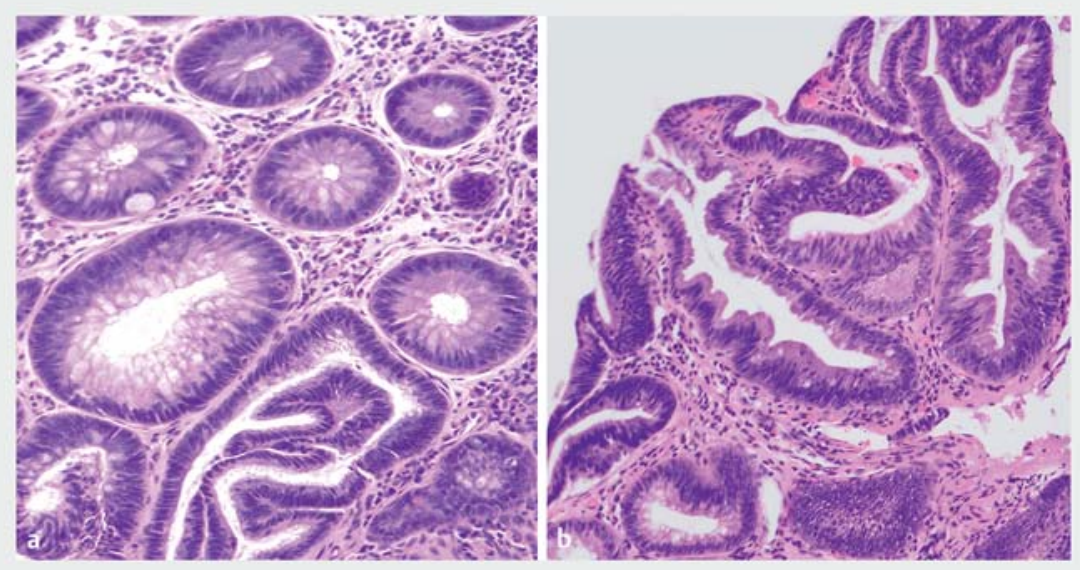

-Fig. 2 Histological examination showed an atypical tubular gland with low-grade dysplasia. a First lesion. b Second lesion.

Competing interests

Shin-ei Kudo, Masashi Misawa, and Yuichi Mori received lecture fees from Olympus Corp. Shin-ei Kudo, Masashi Misawa, and Yuichi Mori have patents (Japan Patent JP 6059271 and JP 6580446) licensed to Cybernet systems and Showa University. Kazuo Ohtsuka reports personal fees and nonfinancial support from Olympus outside the submitted work. Kensaku Mori received a grant from Cybernet Systems.

The authors

Yasuharu Maeda', Shin-ei Kudo', Noriyuki Ogata ${ }^{1}$, Masashi Misawa ${ }^{1}$, Yuichi Mori ${ }^{1}$, Kensaku Mori², Kazuo Ohtsuka ${ }^{3}$

1 Digestive Disease Center, Showa University Northern Yokohama Hospital, Yokohama, Kanagawa, Japan

2 Graduate School of Informatics, Nagoya University, Nagoya, Japan

3 Endoscopy Department, Tokyo Medical and Dental University, Tokyo, Japan
Corresponding author

\section{Yasuharu Maeda, MD}

Digestive Disease Center, Showa University Northern Yokohama Hospital, 35-1

Chigasaki-chuo, Tsuzuki, Yokohama 224-8503, Japan

Fax: +81-45-949-7000

yasuharumaeda610@hotmail.com

\section{References}

[1] Eaden JA, Abrams KR, Mayberry JF. The risk of colorectal cancer in ulcerative colitis: a meta-analysis. Gut 2001; 48: 526-535

[2] Riddell RH, Goldman H, Ransohoff DF et al. Dysplasia in inflammatory bowel disease: standardized classification with provisional clinical applications. Human Pathology 1983; 14: 931-968

[3] Barua I, Vinsard D, Jodal H et al. Artificial intelligence for polyp detection during colonoscopy: a systematic review and metaanalysis. Endoscopy 2020. doi:10.1055/a1201-7165
[4] Misawa M, Kudo SE, Mori Y et al. Artificial intelligence-assisted polyp detection for colonoscopy: initial experience. Gastroenterology 2018; 154: 2027-2029

[5] Misawa M, Kudo SE, Mori Y et al. Development of a computer-aided detection system for colonoscopy and a publicly accessible large colonoscopy video database (with video). Gastrointest Endosc 2020. doi:10.1016/j.gie.2020.07.060

Bibliography

Endoscopy 2021; 53: E273-E274

DOI 10.1055/a-1261-2944

ISSN 0013-726X

published online 1.10 .2020

(c) 2020. Thieme. All rights reserved. Georg Thieme Verlag KG, Rüdigerstraße 14, 70469 Stuttgart, Germany

\section{ENDOSCOPY E-VIDEOS \\ https:/|eref.thieme.de/e-videos}

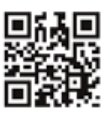

Endoscopy E-Videos is a free access online section, reporting on interesting cases and new

techniques in gastroenterological endoscopy. All papers include a high quality video and all contributions are freely accessible online.

This section has its own submission website at https://mc.manuscriptcentral.com/e-videos 